\title{
Application of Artificial Intelligence Wearable Technology in the Big Data Analysis of Physical Activity in China
}

\author{
Hao Cui ${ }^{1}{ }^{1}$ and Zhiqiang Peng ${ }^{2}$ \\ ${ }^{1}$ Business School, Hunan University of Science and Technology, Xiangtan 411100, Hunan, China \\ ${ }^{2}$ Public Sports Department, Hunan Geely Automobile College, Xiangtan 411100, Hunan, China \\ Correspondence should be addressed to Hao Cui; 1151024@hnust.edu.cn
}

Received 25 August 2021; Revised 11 October 2021; Accepted 18 October 2021; Published 5 November 2021

Academic Editor: Sang-Bing Tsai

Copyright $\odot 2021$ Hao Cui and Zhiqiang Peng. This is an open access article distributed under the Creative Commons Attribution License, which permits unrestricted use, distribution, and reproduction in any medium, provided the original work is properly cited.

\begin{abstract}
Although the validity of the physical activity questionnaire is low, the questionnaire is still the most commonly used measurement tool for physical activity research in China in the past 10 years. In the era of big data, research in the field of physical activity in China needs to be more effective, economical, convenient, and suitable for long-term, large-sample research tools. Acceleration detection technology, heart rate detection technology, and GPS technology are the mainstream technologies for measuring the energy consumption of physical activity in wearable devices. The application of data mining and machine learning methods further enhances the validity of the test. Domestic smart wearable devices are not effective in estimating energy consumption but still have a large space for technical improvement. Smart wearable devices have a very broad application prospect in the field of big data research in physical activity. The impact of smart wearable device technology and big data analysis methods on physical activity research will be far-reaching and may lead to major changes in research concepts, research tools, and data analysis methods.
\end{abstract}

\section{Introduction}

In 1965, one of Intel's founders, Gordon Moore, proposed Moore's Law by long-term observation and summary of the development of computer hardware. The law states that the number of transistors that can be accommodated on an integrated circuit of the same area is approximately one. It will double in 1 2 years. In other words, the processing speed and storage capacity of computer hardware will double every 1 2 years. In fact, since the 1990s, physical memory worldwide has doubled every nine months [1]. Driven by the rapid development of information technology, computers, and the Internet, human society has rapidly entered a new digital age. An era of large-scale production, sharing, and application of data has been launched.

The US Centers for Disease Control and Prevention can monitor new influenza epidemics through a monitoring network setup across the United States, but this data has a one- to two-week delay. In 2009, a study published by Google engineers in Nature found that Google's flu trends obtained through online search records and public health data released 1-2 weeks later are highly consistent, faster, and more economical [2]. Big data has changed our traditional thinking in many fields, such as scientific research, business, and government management. A big change in life, work, and thinking based on big data has quietly occurred.

Big data has a strong impact on all walks of life. How will China's sports research develop in the context of the emergence of big data in many disciplines? Especially, in recent years, with the wide application of wearable devices represented by smart bracelets and smart watches, every change in our physical activity, position and physical physiological data has become data that can be recorded and analyzed. The measurement of activity provides new ideas and methods. There is equipment that can perform real-time analysis of big data in sports activities. Of course, this equipment also has many problems. This is the purpose of our research. We have applied this theoretical possibility to real life $[3,4]$. 
This study will reexamine the current situation of research methods and research tools of physical activity in China from the perspective of big data research, analyze the feasibility of applying smart wearable devices in the research of physical activity big data, and forecast the future development trend.

\section{Related Work}

Studies have shown that the lack of physical activity and the sedentary and static lifestyle are one of the main factors affecting human health [5] and related research on physical activity has become a research hotspot in recent years. However, an important barrier to research related to physical activity is the lack of an accurate, objective, and economical tool for measuring physical activity and static lifestyles. At present, the research methods applied to the measurement of daily physical activity of adolescents and adults mainly include double-standard water method, indirect calorimetry, questionnaire method, heart rate method, and motion sensor method (acceleration meter, pedometer, etc.). The double-standard water method and the indirect calorimetry method have high precision and good stability and are often used as the gold standard for physical activity testing. However, because the two test instruments are expensive, the test cost is high, and the test procedure is complex; they are generally used for small sample experiments and are rarely used for large-scale physical activity studies [6].

The most widely used physical activity measurement tools are a variety of physical activity questionnaires. A research system summarized 130 questionnaires on physical activity, and the reliability level of various physical activity questionnaires ranged from 0.62 to 0.76 , while the validity was only 0.25 to 0.41 , which was not ideal. For example, the international physical activity questionnaire (IPAQ), which is highly recognized and widely used in academia, has a validity of only 0.3 .

Because of the linear relationship between heart rate and energy metabolism in physical activity, some studies have also measured physical activity by monitoring heart rate. However, the correlation between heart rate and energy metabolism has large individual differences, so it is necessary to establish a personalized heart rate-energy relationship before physical activity testing. In recent years, some researchers have developed new algorithms that can estimate energy consumption and assess aerobic endurance levels more accurately without laboratory calibration [7].

Motion sensors, represented by pedometers and accelerometers, are emerging tools for measuring physical activity in recent years. The pedometer estimates the energy consumption of physical activity by recording the number of steps the subject walks. The test cost is economical, but the test accuracy is low. The physical activity monitoring device represented by accelerometer is the most developed tool for measuring physical activity in the past decade. The accelerometer measures the acceleration of human activity through a miniature sensor to estimate the level of physical activity. Since the beginning of the twenty-first century, accelerometers have been gradually applied to the study of physical activity with their excellent validity, and the level of technology has been continuously improved, from the initial single-axis accelerometer to the three-axis accelerometer [8]. Although the price of acceleration sensors is not high due to mass production and application, various types of accelerometers (such as ActiGraph accelerometers) used for scientific research are priced at thousands of dollars, limiting the research on physical activity using accelerometers. Therefore, there are very few large-scale studies of physical activity based on accelerometers in China.

In order to clarify the application of research tools for physical activity in China, this study searched the papers published in the China Knowledge Network database from January 2006 to December 2015 for sports journal articles containing "physical activity" or "physical activity," 223 papers, as shown in Table 1.

In the past 10 years, the number of studies related to physical activity has gradually increased, and the related research on physical activity has received extensive attention from sports scholars. The research tools of 223 papers obtained by the search were classified, and 130 of them were found to be research reviews, theoretical studies, qualitative studies, or methodological studies, and no physical activity measurement tools were used. Of the 82 empirical studies using physical activity measurement tools, 65 used questionnaires, accounting for $79.3 \%$ of all empirical studies, of which the most widely used was the International Physical Activity Questionnaire; about 20\% of the literatures (17) were used. The motion sensor method tests physical activity. Among them, 15 papers use accelerometers and 2 are pedometers. The studies using accelerometers were all published in the period from 2013 to 2015, and the sample size was mostly below 500 .

The innovations of this article are as follows: (1) there is a combination of the old-style questionnaire survey method to do specific research. (2) The research content is related to the theme and does not violate the basic operating common sense. (3) The research methods and research tools used in this article are scientific, effective, convenient, and fast, and special attention is paid to the application of large-scale and efficient data collection tools.

\section{Main Methods of Energy Consumption Measurement of Physical Activity in Smart Wearable Devices}

Under the background of "Internet + " and the Internet of Everything, smart wearable devices with the function of measuring physical activity energy consumption have developed rapidly. Different brands of devices work differently and have different algorithms. The mainstream smart wearable device with physical energy consumption measurement function is developed based on one or more methods of acceleration detection, heart rate detection, and global satellite positioning system (GPS). Wearable technology mainly explores and creates science and technology that can be worn directly on the body or integrated into the 
TABle 1: Common instruments in the measurement of physical activity.

\begin{tabular}{lccc}
\hline & Accuracy & Convenience & Economy \\
\hline Double-standard water & $\star \star \star \star \star$ & $\star \star \star$ & $\star \star$ \\
method & $\star \star \star \star \star$ & $\star \star$ & $\star \star \star$ \\
Indirect calorimetry & $\star \star$ & $\star \star \star \star \star$ & $\star \star \star \star \star$ \\
Questionnaire method & $\star \star \star \star$ & $\star \star \star$ & $\star \star \star$ \\
Heart rate method & $\star \star \star$ & $\star \star \star \star$ & $\star \star \star \star$ \\
Pedometer & $\star \star \star \star$ & $\star \star \star \star$ & $\star \star \star$ \\
Accelerometer method & & &
\end{tabular}

user's clothes or accessories. It is one of the most popular technology types in today's society. Wearable technology is an innovative technology proposed by the Massachusetts Institute of Technology Media Laboratory in the 1960s. This technology can be used to embed multimedia, sensors, and wireless communications into people's clothing and can support gestures and eye movements as a kind of interaction.

\subsection{Estimating the Main Energy Consumption of Physical} Activity Based on Acceleration Detection Technology Method and Basic Principle. Through the "internal connectivity" to achieve rapid data acquisition, through the ultra-fast content sharing ability of efficiently maintaining social connections, get rid of traditional handheld devices and get a seamless network access experience. An intelligent wearable device based on acceleration detection technology detects the acceleration of human body activity through an acceleration sensor and then relies on a series of algorithms to estimate the energy consumption of physical activity of the human body. An acceleration sensor is a sensor that can measure acceleration. It is usually composed of masses, dampers, elastic elements, sensitive elements, and adjustment circuits. Prior to 2009, accelerometer-based physical activity energy consumption algorithms were based primarily on a parameter called "counts" or similar to counts (different companies have different names for such parameters, and algorithms vary). The original signal obtained by the accelerometer is a voltage value that reflects the magnitude of the acceleration. After filtering, amplification, and analogto-digital conversion, the analog voltage signal can be converted into a digital signal, also known as "raw counts," converting this digital signal into counts. Different brands of products use different algorithms; usually there are 3 ways [9]: (1) the number of times the accumulated digital signal exceeds a certain threshold; the threshold can be set to 0 or can be set to represent a certain value of a certain type of exercise or intensity; (2) detect the maximum value in a certain time range to represent the count of the time period; (3) calculate the area under the acceleration data curve in a certain time period, which is also the most commonly used method. A sensor is a device or device that can sense the specified measured and convert it into a usable output signal according to a certain rule. As an important means of information acquisition, sensors, together with communication technology and computer technology, constitute the three pillars of information technology. Using physical, chemical, and biological effects, the measured physical quantity, chemical quantity, biomass, etc. are converted into electricity that meets the needs. In fact, the counts themselves are not meaningful and cannot be understood by people. Usually, a more accurate method of energy metabolism is used as a criterion to establish a regression equation between counts and energy consumption, so that energy consumption can be estimated by counts. This traditional method of estimating the energy expenditure of physical activity has good research validity and is widely used in various types of accelerometers and has also been favored by many physical activity researchers [10].

The traditional algorithm for estimating the energy expenditure of physical activity based on accelerometers is mainly led by a group of researchers in the field of sports science and public health. One of the major drawbacks of estimating the energy consumption of physical activity by counting is to ignore the difference in acceleration characteristics of different types of motion and use the same algorithm to calculate the energy consumption of different types of motion. In addition, the inability of determining the type of exercise is also a major "short board" in the study of physical activity in traditional acceleration calculations $[11,12]$. Since 2009 , especially in the context of the big data era, a group of scientists in computer and mathematics, represented by Bonomi and Staudenmayer, have entered this field of research. They use the data analysis method of big data to conduct a new research on the algorithm of accelerometer estimating energy consumption of physical activity. Through data mining, machine learning, and other data analysis methods, the characteristics of acceleration data of different motion types are analyzed, and the types of motion are used: classification, determining the type, duration, and intensity of the movement, and then establishing energy consumption algorithms based on different types of motion [13]. Some studies have evaluated the new algorithm, further confirming that the new algorithm can identify the type of motion [14] and the energy efficiency of estimating physical activity is superior to the traditional method.

In summary, big data analysis techniques such as data mining and machine learning have become the driving force behind the algorithm revolution that drives accelerometers to estimate physical activity. The establishment of new algorithms is undoubtedly a great advancement in the application of accelerometers to physical activity research and with the continuous improvement of data acquisition and data analysis methods the accelerometer's estimation of energy consumption accuracy may be further improved.

\subsection{The Main Method for Estimating the Energy Expenditure of Physical Activity Based on Heart Rate Detection Technology Law and Basic Principles}

3.2.1. Main Methods for Intelligent Wearable Devices to Detect Heart Rate. In recent years, heart rate testing technology has been continuously improved. There are two main types of heart rate testing techniques for smart wearable devices: (1) ECG measurement technology, the subject needs to wear a wireless heart rate chest strap, which records the 
heartbeat. The bioelectrical signal measures the heart rate and its principle is similar to the electrocardiogram test. The method has the advantages of high test accuracy and good test stability under motion; the disadvantage is that the heart rate chest strap needs to be worn on the chest and the user's comfort may be affected, which is not conducive to the user's long-term continuous use. (2) Photoelectric testing technology: the basic principle of this technology is that the blood in the blood vessels of the arm will change the hemoglobin absorbance when the pulse is pulsating. By detecting the change of the light transmittance, the pulse can be tested. The advantage of this test technology is that you do not need to wear a chest strap. Users only need to wear related equipment on the arm or wrist, which helps to improve the "user's stickiness." The disadvantage is that the test accuracy is lower than the ECG test. The stability in the state is also slightly worse. Even so, optoelectronic testing technology has been favored by more manufacturers for its superior comfort and user stickiness.

\subsubsection{Algorithm for Estimating Heart Rate-Energy} Consumption. Applying heart rate to estimate energy expenditure has been the focus of researchers for decades ago. In the 1950s, Berggren et al. established an estimation method based on the near linear relationship between heart rate and oxygen uptake and energy expenditure in submaximal motion. Based on this linear relationship, the submaximal maximum oxygen uptake test method, which is very important in the measurement and evaluation of aerobic endurance, was established. However, there are some limitations in estimating heart rate-energy consumption: (1) heart rate 82 is vulnerable to emotions; (2) when energy consumption is low, heart rate estimation energy consumption error is large; (3) everyone's heart rate is different. There is a big difference in heart rate response between different exercise loads; that is, there is a large individual difference in the relationship between heart rate and energy expenditure. The standard heart rate method estimates energy consumption and requires a "calibration" in the laboratory to determine the linear relationship between heart rate and energy expenditure for the subject. However, it is unrealistic and inconvenient for users of wearable devices to go to the lab for testing. Therefore, the estimated errors of various smart wearable devices that use the heart rate method to estimate energy consumption are quite different from individualized differences. In recent years, many researchers are exploring new ways to reduce individual errors and improve heart rate estimation energy consumption through a number of different techniques (big data analysis, indirect personal calibration, etc.) [15]. The practical effects of these technology applications are still to be further studied, and they are currently only applied to certain brands on a small scale and have not been adopted by most smart wearable devices.

3.3. Estimating the Energy Consumption of Physical Activity Based on the Global Satellite Positioning System Method and Basic Principle. The Global Positioning System (GPS) is a satellite-based and communication-based positioning system that estimates the position, speed (walking or running), slope (by altitude or map), and time of the subject during outdoor sports. Speed and slope are the two main factors in the estimates of oxygen uptake and energy consumption recommended by the American College of Sports Medicine (ACSM). Some researchers used accelerometers as a benchmark to compare the effectiveness of energy consumption measured by three brands of GPS watches. It was found that the estimated energy consumption of GPS and the accelerometer measurement were $40 \%$ to $50 \%$ differently. Current research suggests that GPS has a greater error in measuring high-speed motion of the subject and increasing the GPS sampling frequency may help to improve the effectiveness of the measurement. One problem with GPS measurement of energy expenditure in physical activity is that it is impossible to measure physical activity in the room; even if it is applied to outdoor sports, it is affected by some environmental factors (buildings, trees, clouds, etc.). Despite some problems, GPS can be combined with other methods to increase the effectiveness and reliability of energy activity estimates for physical activity.

\section{Feasibility of Applying Smart Wearable Devices to Physical Activity Big Data Research}

In the new era of "Internet +" and the Internet of Everything, smart wearable devices represented by smart sports bracelets and smart sports watches have developed rapidly. The mainstream smart wearable devices for physical activity monitoring are developed based on one or more technologies such as three-axis acceleration sensors, heart rate sensors, gyroscopes, and GPS positioning. In view of accelerometers, based on accelerometers, such as ActiGraph accelerometers and RT3 accelerometers, domestic and foreign studies have proven that they can accurately measure the energy consumption of physical activity and have been widely used in the study of various physical activities, and many smart wearable devices are similar to the ActiGraph accelerometer and RT3 accelerometer. The accelerometers are similar, and many smart wearable devices are equipped with heart rate, GPS, gyroscope, and other technologies. Therefore, in theory, the accuracy of smart wearable devices for testing physical activity should be higher or at least comparable to accelerometers.

According to the Nyquist Criterion, the sampling frequency of physical activity measurement should be at least 2 times the highest frequency of motion, and the sampling frequency of most commercial physical activity monitoring and recording equipment is $1 \sim 64 \mathrm{~Hz}$. As a result, smart wearables record that the amount of data acquired by physical activity is quite large and has a very fast data growth rate. In addition, as mentioned earlier, information such as motion type, exercise intensity, and motion time can be provided through some data mining techniques. If you use the GPS function, you can also use the motion position and time to get a lot of relevant data in combination with geographic information, weather forecast information, traffic information, air quality information, and so on. The 
rich data types have expanded the thinking for future physical activity big data research, and many studies that were difficult to carry out in the future may be realized in the future.

With the rapid development and maturity of smart wearable technology, many smart sports bracelets using three-axis accelerometers, for example, have been sold at a lower price, further expanding the size of the population. In addition, the smart wearable device can realize remote data transmission through the mobile terminal and return uninterrupted massive personal activity data to the researcher's server through the Internet, and the data collection work is more convenient and quick. Therefore, smart wearable products, if applied to large-scale research on physical activity, will be very economical and easy to use. Epson released the Pulsense series of wearable devices, including smart watches and smart bracelets. These products integrate Epson's industry-leading original biosensing technology and cloud-based services to meet the fitness, health, and sports needs of the consumer wearable market.

In summary, the data provided by the smart wearable system have the characteristics of large capacity, fast data growth, and data diversity. The equipment also has excellent economics and ease of use, laying the foundation for future large-scale physical activity big data research using smart wearable devices.

\section{Study on the Effectiveness of Existing Domestic Smart Wearable Devices for Measuring Physical Activity}

As the most basic smart wearable devices, the intelligent sports bracelet is objective and meticulous (data acquisition can be accurate to $\mathrm{s}$ or $\mathrm{ms}$ ), having large amount of data, rich, convenient, and economical, compared with the physical activity questionnaire (Table 2). Traditional intelligent sports bracelets cannot automatically distinguish motion types, but, in recent years, related algorithms have matured, and motion types can be automatically recognized and have good effectiveness. However, the effectiveness of domestic smart wearable devices to test physical activity energy consumption has rarely been reported. Therefore, this study studies the effectiveness of energy consumption of physical activity based on the laboratory environment and a smart bracelet based on daily life environment to explore the effectiveness of the domestic smart bracelet in measuring the energy expenditure of physical activity in the application scenario.

\subsection{Experiment 1: Measuring the Physical Activity Energy of} the Smart Bracelet Based on the Laboratory Environment Volume Consumption Effectiveness Study. The study subjects were 36 adults, 18 males and 18 females; the youngest age was 22 years old and the maximum age was 27 years old. All subjects were healthy and had no respiratory distraction, cardiovascular and cerebrovascular diseases, and endocrine systems. The four types of smart bracelets that require a large number of domestic users are required: $\mathrm{H}$-ring, X-Brace,
B-Brace, and G-Brace. Subjects walked or ran for $3 \mathrm{~min}$ on a laboratory treadmill at speeds of $4.0 \mathrm{~km} / \mathrm{h}, 5.6 \mathrm{~km} / \mathrm{h}, 6.4 \mathrm{~km} /$ $\mathrm{h}, 7.2 \mathrm{~km} / \mathrm{h}$, and $8.0 \mathrm{~km} / \mathrm{h}$, respectively. The energy consumption of the human body at each speed was measured using four sports bracelets and a COSMED Quark gas analysis mass spectrometer. The energy consumption measured by indirect calorimetry is used as a criterion to compare the measured values of different smart bracelets.

As shown in Table 3, different brands of smart bracelets measure the energy consumption of physical activity and the energy consumption measured by indirect calorimetry has different degrees of error; generally there is an underestimation of energy consumption; the average error ranges from $2 \%$ to $40 \%$.

The correlation coefficient of energy consumption measured by different brands of smart bracelets and gas analysis ranges from 0.02 to 0.67 . Some brands measure the effectiveness of energy consumption at certain speeds in an acceptable range. However, individual brands' intelligent bracelet energy consumption data is almost irrelevant to indirect calorimetry data, and measurement energy consumption is less effective. Using the speed and slope-based American Sports Medicine Association (ACSM) to calculate the energy calculation formula for walking and running, it is found that the correlation coefficient between the estimated energy consumption and the measured value using the ACSM formula is 0.62 to 0.75 and the statistical tests are significant (Table 4).

\subsection{Experiment 2: Measuring Physical Activity Based on the} Smart Bracelet of Daily Life Environment Energy Consumption Effectiveness Study. The study subjects were 18 primary school students aged 10 to 12,7 boys and 11 girls. The subjects were healthy and did not have any diseases that were not suitable for exercise. Subjects were asked to wear the ActiGraph accelerometer and a brand of smart sports bracelet for 7 days in a row, except for special circumstances such as bathing or swimming, without taking off the wearing equipment. The energy consumption measured by the ActiGraph accelerometer, which is widely used in physical activity research, is a calibration to calculate the effectiveness and measurement error of the intelligent sports bracelet test.

In the real-life environment, the intelligent sports bracelet and ActiGraph accelerometer measured the average daily physical activity caused by energy consumption of $259.2 \pm 171.9 \mathrm{kcal}$ and $274.4 \pm 139.0 \mathrm{kcal}$, with a correlation coefficient of 0.66 and a measurement error of $15.7 \%$.

\subsection{Discussion on Measurement Validity of Domestic Smart} Wearable Devices. The data of this study show that the test results of indirect calorimetry in the laboratory environment are the calibration and the validity of different brands of domestic intelligent sports bracelets estimated at different walking or running speeds is very different. In general, although the test validity and accuracy of the national smart bracelet are not high, the test validity is still higher than the validity of various physical activity questionnaires $(0.24 \sim 0.41)$. It should be pointed out that, compared with 
TABLE 2: Comparison of physical activity questionnaire and smart wearable devices.

\begin{tabular}{lcc}
\hline & Questionnaire & Smart wearable device \\
\hline Data collection & Subjective and fuzzy & Objective and meticulous \\
The amount of data & Less and single & Much and rich \\
Practicality & Convenient and economical & Convenient and economical \\
Type of exercise & Measurable & Recognizable \\
Validity & $0.25 \sim 0.41[1]$ & Unknown \\
\hline
\end{tabular}

TABLE 3: Comparison of the energy expenditure measured by four types of smart bracelets and indirect calorimetry.

\begin{tabular}{|c|c|c|c|c|c|c|c|c|c|}
\hline \multirow[b]{2}{*}{$\begin{array}{l}\text { Speed } \\
(\mathrm{km} / \mathrm{h})\end{array}$} & \multicolumn{2}{|c|}{$\mathrm{H}$ wristband } & \multicolumn{2}{|c|}{$\mathrm{X}$ wristband } & \multicolumn{2}{|c|}{ B wristband } & \multicolumn{2}{|c|}{ G wristband } & \multirow[b]{2}{*}{$\begin{array}{l}\text { Indirect calorimetry } \\
\text { energy } \\
\text { consumption (kcal) }\end{array}$} \\
\hline & $\begin{array}{c}\text { Energy } \\
\text { consumption } \\
(\mathrm{kcal})\end{array}$ & $\begin{array}{c}\text { Error } \\
(\%)\end{array}$ & $\begin{array}{c}\text { Energy } \\
\text { consumption } \\
(\mathrm{kcal})\end{array}$ & $\begin{array}{c}\text { Error } \\
(\%)\end{array}$ & $\begin{array}{c}\text { Energy } \\
\text { consumption } \\
(\mathrm{kcal})\end{array}$ & $\begin{array}{c}\text { Error } \\
(\%)\end{array}$ & $\begin{array}{c}\text { Energy } \\
\text { consumption } \\
(\mathrm{kcal})\end{array}$ & $\begin{array}{c}\text { Error } \\
(\%)\end{array}$ & \\
\hline 4.0 & 18.61 & 19.60 & 15.00 & -3.60 & 11.33 & $\begin{array}{c}- \\
27.19\end{array}$ & 15.19 & -2.38 & 15.56 \\
\hline 5.6 & 19.75 & -7.45 & 19.03 & $\begin{array}{c}- \\
10.82\end{array}$ & 14.61 & $\begin{array}{c}- \\
31.54\end{array}$ & 19.03 & $\begin{array}{c}- \\
10.82\end{array}$ & 21.34 \\
\hline 6.4 & 22.19 & $\begin{array}{c}- \\
16.70\end{array}$ & 22.86 & $\begin{array}{c}- \\
14.19\end{array}$ & 18.58 & $\begin{array}{c}- \\
30.26\end{array}$ & 20.67 & 22.41 & 26.64 \\
\hline 7.2 & 29.31 & $\begin{array}{c}- \\
16.99\end{array}$ & 31.69 & $\begin{array}{c}- \\
10.25\end{array}$ & 30.92 & $\begin{array}{c}- \\
12.43\end{array}$ & 23.64 & $\begin{array}{c}- \\
33.05\end{array}$ & 35.31 \\
\hline 8.0 & 40.39 & -2.91 & 41.53 & -0.17 & 47.47 & 14.11 & 24.92 & $\begin{array}{c}- \\
40.10\end{array}$ & 41.60 \\
\hline Overall & 130.25 & -7.27 & 130.11 & - 7.37 & 122.94 & $\begin{array}{c}- \\
12.47\end{array}$ & 103.31 & $\begin{array}{c}- \\
26.45\end{array}$ & 140.46 \\
\hline
\end{tabular}

TABLE 4: Correlation coefficients in the energy expenditure measured by four types of smart bracelets, ACSM equations, and indirect calorimetry.

\begin{tabular}{lccccc}
\hline Speed $(\mathrm{km} / \mathrm{h})$ & H wristband & X wristband & B wristband & G wristband & 0.11 \\
ACSM formula \\
\hline 4.0 & $0.37^{*}$ & 0.20 & 0.04 & -0.05 & $0.62^{* *}$ \\
5.6 & $0.67^{* *}$ & $0.37^{*}$ & -0.16 & 0.14 & $0.69^{* *}$ \\
6.4 & $0.58^{* *}$ & $0.34^{*}$ & -0.02 & -0.25 & $0.73^{* *}$ \\
7.2 & 0.11 & 0.15 & -0.30 & -0.11 & $0.75^{* *}$ \\
8.0 & 0.29 & $0.39^{*}$ & -0.13 & $0.74^{* *}$ \\
\hline
\end{tabular}

${ }^{*}$ indicates a statistical difference; ${ }^{* *}$ indicates a significant statistical difference.

the average error of $9.3 \% \sim 23.5 \%$ of some brands in foreign research reports and the correlation coefficient of $0.71 \sim 0.93$, the overall level of domestic intelligent sports bracelet measurement is still relatively different. The main reasons for the inaccuracy of the energy consumption test of the domestic smart bracelet may be as follows:

(1) Limitations of some intelligent sports bracelet algorithms: the experiment in the study was completed on the treadmill of the laboratory. The acceleration of the subject in the direction of the sagittal axis was different from that of the outdoor walking and running, which led to a large error in the estimated value of some sports smart bracelets. In the daily life environment, the test validity and accuracy of the domestic intelligent sports bracelet are greatly improved compared with the laboratory. It is suggested that the algorithm of the domestic intelligent sports bracelet may have limitations and is not suitable for the treadmill environment. The algorithm needs to be further improved.
(2) The impact of smart wearable device wearing position on energy consumption estimates: the wearable device selected for the study was a smart bracelet that was worn at the wrist away from the torso. As the smart wearable device based on the accelerometer is closer to the torso, the measured physical activity represents the whole body motion, and the accuracy of the test is higher. Therefore, the smart sports bracelet is worn on the wrist and located at the distal end of the upper limb. During the test, some local movements of the upper limb are easily recognized by the smart bracelet as whole body motion, thereby affecting the test results.

(3) The algorithm for energy consumption of intelligent sports bracelets relies too much on acceleration sensor data. Although some manufacturers have introduced a smart version of the smart sports bracelet, heart rate indicators have not been used in energy consumption algorithms for a variety of reasons. It must be pointed out that there are still 
some shortcomings in the energy consumption algorithm relying solely on the acceleration sensor. For example, slope is an important factor affecting energy consumption. The energy consumption of uphill walking and walking is very different. According to ACSM's energy consumption calculation formula, compared with the same speed flat running, a slope of $10 \%$ can be used increasing energy consumption by nearly $45 \%$. However, most smart bracelets do not have a slope recognition function, and the estimation of energy consumption is greatly affected. Studies have confirmed that combining different testing techniques may help to improve the estimated validity of energy consumption.

(4) The choice of algorithm technology: as mentioned earlier, studies have shown that new algorithms based on data mining and machine learning are more effective than traditional algorithms. In order to further understand the current mainstream smart bracelet algorithm, this study selected the mainstream smart sports bracelets sold in the market according to the sales data of the mainstream network e-commerce sales platform and investigated 38 kinds of products by consulting the product manual and the product on-site trial. Bracelet: Amiigo, Amazfit, Bithealth, Bong, Capshi, Dido, Fitbit, Gamin, Gisso, Isport, JDHDL, Jawbone, Mate, Misfit, Moto, Nike, Polar, Sony, Ticwatch, 360, 37 Feike, Huawei, Le Run, Lexin, Lei Snake, Ai Wei, Quancheng Tong, Samsung, Brush, Newman, Wei Le, Yi Wei, Xiaomi, Yi Qingteng, Yu Jie, and Tian Sheng. The survey found that only five types of bracelets have automatic motion recognition and the remaining 33 bracelets do not have this function and do not have the basis for adopting new algorithmic techniques. The slow update speed of algorithm technology may be one of the important reasons for the low validity of physical activity measurement.

\section{Rethinking the Research Methodology of Physical Activity in China under the Background of Big Data Era}

\subsection{Is It Accurate or Fuzzy? Rethinking on the Research} Concept. In the traditional theory of physical measurement and evaluation and sports statistics, the reliability and effectiveness of measurement tools are the indicators that researchers focus on when selecting research tools. In order to make the research results more accurate, many researchers have been working on the optimization of measurement tools. In the era of big data, large-capacity, highspeed growth and diversified data have enabled researchers to see the whole time, full-coverage as a whole, reducing the embarrassment of "blind-eyed elephants" and "blind people" that may arise due to small data research. Of course, the pursuit of breadth and frequency of data sometimes has to make some sacrifices and compromises on the accuracy of the data. The $24 \mathrm{~h}$ ECG measurement accuracy is not as good as the normal 12-lead ECG, but it provides a wider timedomain and larger capacity ECG data, which is still very meaningful. Therefore, in the big data research of physical activity, if you can get a wider time-domain (lasting days or even months), higher frequency (data frequency reaches s or even ms level), and more types through smart wearable devices (physical activity data of exercise type, exercise intensity, exercise time, exercise location, surrounding environment, physiological response, etc., appropriate sacrifice of measurement accuracy of some data may be a wise compromise. From this perspective, even though the current testability of smart wearable devices is not ideal, it may not hinder its application in physical activity research.

6.2. Is the Questionnaire Still a Smart Wearable Device? Rethinking about Research Tools. For a long time, the physical activity questionnaire has been one of the most commonly used tools for studying physical activity at home and abroad. The results of this study also show that $79.3 \%$ of the empirical research tools in the field of physical activity are physical activity questionnaires. Questionnaires are relatively economical and simple measurement tools and have been widely used in large-scale physical activity research in recent years. However, the questionnaire also has problems such as strong subjectivity and low measurement validity. With the rapid rise of wearable devices represented by smart sports bracelets, smart wearable devices with higher validity, more rich data, easier to use, and more economical cost may bring an upgrade to the physical activity research tool. Big data research based on smart wearable devices may become the mainstream of future physical activity research.

The technology of intelligent wearable devices applied to physical activity research is still in the exploratory stage, and the low measurement validity also limits its promotion in professional research fields. Therefore, smart wearable devices are more often positioned as "sounds" for sports enthusiasts than research tools for professional researchers. However, in the early 19th century, when the train was just invented, it was ridiculed because it was slower than the carriage. Similarly, people should not give up because some domestic brands of smart bracelets are not ideally valid for the time being. On the contrary, smart wearable devices have a very large room for improvement and have a very broad space for development in the future of big data research on physical activity in China and application prospects.

\subsection{Analysis of Variance or Neural Network? Rethinking on} Data Analysis Methods. The traditional method of data statistics is based on "small data," and "big data" itself has the characteristics of complex data structure, huge volume, and latent value. In the vast amount of data, the relationship between variables is sought. The traditional statistical methods cannot meet the requirements. The need for data analysis, therefore, requires newer, better statistical methods. In this context, new data analysis methods such as data mining and machine learning have been rapidly developed. Data mining and machine learning analysis methods represented by neural networks and decision trees 
are used in automatic identification of physical activity types and energy consumption estimation. The application has achieved fruitful results, and these are difficult problems to be solved by traditional statistical analysis methods such as $t$ test and analysis of variance. If big data research is the future direction of physical activity, then data mining and machine learning may replace traditional data analysis methods in the near future, becoming the mainstream method of data analysis in physical activity research.

\section{Conclusion}

After analyzing and comparing all the above content, we can basically draw research tools suitable for the research needs of the field of sports activities and also suitable for the development needs of the times. On this basis, this type of big data analysis tools also has the advantages of previous questionnaire surveys.

Acceleration detection technology, heart rate detection technology, and GPS technology are the mainstream technologies for measuring the energy consumption of physical activity in wearable devices. The application of data mining and machine learning methods further enhances the validity of the test. However, we still need to note that despite the fact that there are a lot of research foundations, China's current wearable devices still have many shortcomings. The most obvious is that they are not sure about the specific energy consumption value. We do not need to worry too much, because relevant research is not uncommon. Smart wearable devices have a very broad application prospect in the field of big data research in physical activity.

The impact of smart wearable device technology and big data analysis methods on physical activity research will be far-reaching and may lead to major changes in research concepts, research tools, and data analysis methods.

\section{Data Availability}

No data were used to support this study.

\section{Conflicts of Interest}

The authors declare that they have no conflicts of interest.

\section{References}

[1] Q. Mu, T. Wang, and Y. Jia, "Research on framework of underground wearable devices framework based on cloud computing," in Proceedings of the 2015 8th International Symposium on Computational Intelligence and Design (ISCID), pp. 458-461, Hangzhou, China, 2015.

[2] A. Leadbetter, D. Smyth, R. Fuller, E. O'Grady, and A. Shepherd, "Where big data meets linked data: applying standard data models to environmental data streams," in Proceedings of the 2016 IEEE International Conference on Big Data (Big Data), pp. 2929-2937, Washington, DC, USA, 2016.

[3] Z. Lv, A. Halawani, S. Feng, S. Ur Réhman, and H. Li, "Touchless interactive augmented reality game on vision-based wearable device," Personal and Ubiquitous Computing, vol. 19, no. 3-4, pp. 551-567, 2015.
[4] Z. Lv, R. Lou, J. Li, A. K. Singh, and H. Song, "Big data analytics for 6G-enabled massive Internet of Things," IEEE Internet of Things Journal, vol. 8, no. 99, p. 1, 2021.

[5] Q. Cheng, Z. Peng, J. Lin, S. Li, and F. Wang, "Energy harvesting from human motion for wearable devices," in Proceedings of the 10th IEEE International Conference on Nano/ Micro Engineered and Molecular Systems, pp. 409-412, Xi'an, China, 2015.

[6] E. Mohamed, "The relation of artificial intelligence with Internet of things: a survey," Journal of Cybersecurity and Information Management, vol. 1, no. 1, pp. 30-24, 2020.

[7] B. Li, X. Ming, and G. Li, "Big data analytics platform for flight safety monitoring," in Proceedings of the 2017 IEEE 2nd International Conference on Big Data Analysis (ICBDA), pp. 350-353, Beijing, China, 2017.

[8] C. F. Xu, F. B. Tao, Y. F. Gong, J. Cao, and X. W. Wang, "Substation inspection based on wearable devices and big data technology," in Proceedings of the International Conference on Renewable Power Generation (RPG 2015), pp. 1-5, Beijing, China, 2015.

[9] R. Tardío, A. Mate, and J. Trujillo, "An iterative methodology for big data management, analysis and visualization," in Proceedings of the 2015 IEEE International Conference on Big Data (Big Data), pp. 545-550, Santa Clara, CA, USA, 2015.

[10] L. Zhu, H. Hwang, E. Ren, and G. Yang, "High performance MIMO antenna for 5G wearable devices," in Proceedings of the 2017 IEEE International Symposium on Antennas and Propagation \& USNC/URSI National Radio Science Meeting, pp. 1869-1870, San Diego, CA, USA, 2017.

[11] S. Wan, L. Qi, X. Xu, C. Tong, and Z. Gu, “Deep learning models for real-time human activity recognition with smartphones," Mobile Networks and Applications, vol. 25, pp. 743-755, 2019.

[12] H. Song and M. Brandt-Pearce, "A 2-D discrete-time model of physical impairments in wavelength-division multiplexing systems," Journal of Lightwave Technology, vol. 30, no. 5, pp. 713-726, 2012.

[13] S. Cho, S. Hong, and C. Lee, "ORANGE: spatial big data analysis platform," in Proceedings of the 2016 IEEE International Conference on Big Data (Big Data), pp. 3963-3965, Washington, DC, USA, 2016.

[14] T. H. A. S. Siriweera, I. Paik, B. T. G. S. Kumara, and K. R. C. Koswatta, "Intelligent big data analysis architecture based on automatic service composition," in Proceedings of the 2015 IEEE International Congress on Big Data, pp. 276-280, New York, NY, USA, 2015.

[15] Y. Sun, Y. Tao, Z. Hu, H. Fan, and Y. Wang, "A hands-free communication solution for wearable devices," in Proceedings of the 2014 IEEE Healthcare Innovation Conference (HIC), pp. 75-78, Seattle, WA, USA, 2014. 